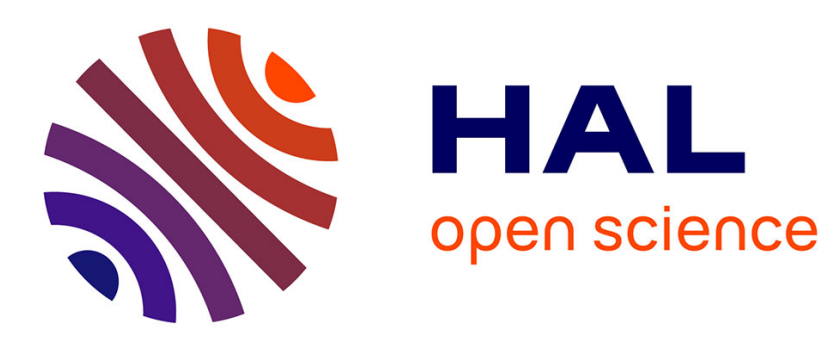

\title{
Setup and Troubleshooting of Volta Phase Plate Cryo-EM Data Collection
}

Ottilie von Loeffelholz, Bruno Klaholz

\section{To cite this version:}

Ottilie von Loeffelholz, Bruno Klaholz. Setup and Troubleshooting of Volta Phase Plate Cryo-EM Data Collection. Structural Proteomics, 2305, Springer US, pp.291-299, 2021, Methods in Molecular Biology, 10.1007/978-1-0716-1406-8_14 . inserm-03376627

\section{HAL Id: inserm-03376627 https://www.hal.inserm.fr/inserm-03376627}

Submitted on 6 Dec 2021

HAL is a multi-disciplinary open access archive for the deposit and dissemination of scientific research documents, whether they are published or not. The documents may come from teaching and research institutions in France or abroad, or from public or private research centers.
L'archive ouverte pluridisciplinaire HAL, est destinée au dépôt et à la diffusion de documents scientifiques de niveau recherche, publiés ou non, émanant des établissements d'enseignement et de recherche français ou étrangers, des laboratoires publics ou privés. 


\section{Setup and troubleshooting of Volta phase plate cryo-EM data collection}

Ottilie von Loeffelholz ${ }^{1,2,3,4}$, Bruno P. Klaholz ${ }^{1,2,3,4^{*}}$

${ }^{1}$ Centre for Integrative Biology (CBI), Department of Integrated Structural Biology, IGBMC (Institute of Genetics and of Molecular and Cellular Biology), 1 rue Laurent Fries, Illkirch, France,

${ }^{2}$ Centre National de la Recherche Scientifique (CNRS) UMR 7104, Illkirch, France,

${ }^{3}$ Institut National de la Santé et de la Recherche Médicale (Inserm) U964, Illkirch, France,

${ }^{4}$ Université de Strasbourg, Strasbourg, France

*To whom correspondence should be addressed: klaholz@igbmc.fr

Tel.: 0033.369.48.52.78 


\begin{abstract}
Cryo electron microscopy (cryo-EM) has become a method of choice in structural biology to analyse isolated complexes and cellular structures. This implies adequate imaging of the specimen and advanced image processing methods to obtain highresolution 3D reconstructions. The use of a Volta phase plate in cryo-EM drastically increases the image contrast while being able to record images at high acceleration voltage and close to focus, i.e. at conditions where high-resolution information is preserved best. Higher contrast images behave better during image processing because they can be better aligned and classified resulting in lower data amount requirement and increased data quality. Here we give step-by-step guidelines how to set up high quality VPP cryo-EM single particle data collections, as exemplified by human ribosome data acquired during a one day data collection session. Further, we describe specific technical details in image processing that differ from conventional single particle cryoEM data analysis.
\end{abstract}




\section{Key words}

Volta phase plate, phase contrast, cryo electron microscopy, human ribosome, structural biology

Running head:

VPP cryo-EM data collection 


\section{Introduction}

Visualisation of biomolecules relies on good image contrast, which in cryo electron microscopy (cryo-EM) is almost entirely achieved by phase contrast. Macromolecular complexes are weak phase objects resulting in very low contrasted images when they are imaged in focus (1). Changes in imaging, such as the usage of lower voltage $(2,3)$ and defocussing (4) are traditionally implemented to generate higher contrast. Insertion of a phase plate in the back-focal plane instead of an objective aperture (Fig. 1) also allows imaging at high contrast with the advantage that imaging can be done at high voltage and very close to focus, typically two parameters that help acquiring strong high frequency components, i.e. the high-resolution data. This can be achieved by an additional negative phase shift between the sample-scattered and the un-scattered electrons which results in a drastic increase of the sample-scattered image intensities. Various phase plate approaches (5-9) have been tried in the past, but the one that now allows routine usage for data collection both for single particle cryo-EM and cryo electron tomography (cryo-ET) is the Volta phase plate (VPP) (10).

The VPP is constituted of a thin carbon film placed in the back-focal plane of the optical system; exposure to the electron beam induces a phase shift which increases with the exposure time, i.e. it is an effect building up under cumulative dose (10). Phase shifts from $\sim 45-135$ degree were shown to be optimal for image processing (11), implying that the phase plate position needs to be changed on a regular basis when the phase shift becomes too high (e.g. after 1-2h or every 100-150 images, depending on imaging settings). The relatively easy handling, reproducibility and low level of artefacts makes the VPP currently superior to other phase plate developments, such as the Zernike phase plate $(6,12,13)$ and electrostatic phase plates $(5,7,9)$, but there are 
nevertheless specific aspects to be taking care of on the electron microscope before starting a data collection (e.g. constant heating temperature, check for low contamination, VPP centering, etc. as detailed below). When a VPP is used, the increase in contrast correlates with an increasing phase shift. After a phase shift of $\sim 90$ degrees the low resolution information takes overhand leading to increased image blurring (11). It needs to be mentioned that the estimation of the contrast transfer function $(\mathrm{CTF})$ is not yet well understood at phase shifts beyond 180 degrees. Therefore, even though it was suggested that high resolution structures can be obtained from data including high phase shift images (14) it is not advised to use images with a phase shift higher than 180 degrees. Additionally, it is advisable to collect VPP cryo-EM data with a small defocus (11), even though in-focus (11) and even over-focus data (15) collections were shown to be usable in image processing leading to structures in the $3 \AA$ range. Defocus VPP data collection is beneficial in terms of i) automatically setting up the defocus target in the microscope, ii) estimation of the correct CTF and iii) speed of data collection $(11,16)$. The huge advantage of using the VPP for single particle data collection is the high contrast obtained that helps aligning the particles $(10,11,16)$ so that structures of proteins down to a size of $50-80 \mathrm{kDa}(17-19)$ can be solved to $3-4 \AA$ resolution by single particle image processing approaches (provided sample homogeneity and particle distribution etc. are good). It also facilitates 2D particle classifications and 3D classifications of the reconstructions using various structure sorting approaches (20-23), structure refinement with focused classifications and refinements $(24,25)$, and it facilitates cryo-EM map interpretation and atomic model building (26). The VPP is a promising tool also for acquiring cryo electron tomograms to increase the visibility of 
otherwise too noisy samples, which allows better segmentation and interpretation of the data $(27,28)$.

In this article we describe a hands-on protocol for setting up a successful VPP cryo-EM data collection session including practical hints on how to overcome difficulties that can occur during alignment of the VPP for data collection and for CTF estimation during image data processing. 


\section{Materials}

2.1 Cryo-EM grids suitable for single particles data collection. These can be for example Quantifoil or C-flat grids with regular hole sizes and regular spacing in between them. Irregular grids (e.g. Lacy) are also possible but will reduce throughput and ease of setting up automated data collection.

2.2 A cryo electron microscope equipped with a VPP, which is inserted instead of an objective aperture. In our case a Titan Krios electron microscope (Thermo Fisher Scientific) is used.

2.3 Software for automated data collection such as EPU (Thermo Fisher Scientific) or SerialEM (29)

2.4 A GPU machine with programs for data processing installed (here: GCTF, Motioncor2, Excel or OpenOffice)

\section{Methods}

The VPP is very sensitive mechanically and with respect to the electron beam and it can contaminate easily. It needs to be removed every time when changing the grid in the microscope to avoid contamination of the VPP. Additionally, to avoid contamination of the VPP during data collection the heating current of the VPP should be set at around 25 $\mathrm{mA}$ to achieve a stable temperature of $\sim 200^{\circ} \mathrm{C}$, and the heating device should remain on to obtain a stable temperature; in case of vacuum failure of the microscope the heating should be switched off to avoid oxidation of the VPP carbon foil.

1. Go to Eucentric height and desired recording magnification and set Eucentric focus. Choose the $50 \mu \mathrm{m}$ condenser aperture. 
2. Perform direct alignments procedure including beam pivot points, beam shift, rotation centre on a Cross Grating grid.

3. Move to an empty area on the grid and decide about the Spot size used for data collection. Insert the phase plate (instead of an objective aperture). Insert the fluorescence screen.

4. Bring the phase plate in the back-focal plane. Therefore tick the box "MF-Y fine focus back-focal plane" in the phase plate tab and turn the MF-Y button until a minimum of artefacts are seen, e.g. stripy or cloud-like shades (close to the back-focal plane) or white or black contamination spots (far from the back-focal plane; Figure 1, see Note 1).

5. Additional condenser astigmatism (Figure 1) can be removed by ticking the condenser box in the apertures tab additionally to the "MF-Y fine focus back-focal plane" box and turning MF-X and MF-Y buttons.

6. Go to the Alignments tab and chose "Align PhasePlate" and then "Phase Plate $\mu$ P: microprobe mode". Perform all steps by following all the instructions (see Note 2). Then move on to "Phase Plate nP: nanoprobe mode" and perform all steps following the instructions. This procedure sets the diffraction lens value that focuses on the phase plate and also sets accurate beam shift pivot points on the phase plate (see Note 3), so that the same position of the VPP is exposed in focus and exposure settings, which is important to keep the phase shift relatively similar. The alignments should be repeated until the settings do not need to be changed anymore.

7. Retract the phase plate.

8. If present, perform a full alignment of the Quantum energy filter in suitable software. 
9. In case the microscope contains a Cs corrector: go to eucentric height on a flat area of the grid and use the image corrector software to align the corrector.

10. If necessary, realign the rotation centre again.

11. Acquire Gain and Dark references on an empty grid square.

12. Change the grid to the specimen grid and acquire a grid map in low magnification.

13. Choose suitable areas for data collection in the grid map, ensure the image shift is minimal and acquire medium magnification images of the grid squares.

14. Select suitable areas for data collection, decide about the maximum number of images to be acquired per hole and define focussing area.

15. Go to an empty area on the grid, choose the spot size for imaging. Decide about the target dose and fractionation scheme, insert the phase plate and repeat steps 3 and 4 immediately before starting the data collection.

16. Take an image in record settings to ensure that there are no artefacts created by the VPP (these can be recognized from shades over the image of an empty grid square (Fig. 1) or uneven contrast of particles inside the image (Figure 2D and E)).

17. Move to an area with carbon and correct the astigmatism induced by the phase plate with the objective stigmator.

18. Move to the next position of the VPP.

19. Start automated data collection with only one fixed defocus (e.g. $-500 \mathrm{~nm})$ or a small target defocus range (differing only by $-200--600 \mathrm{~nm}$ ) and focussing at every stage position (see Note 4,5). Here, the difference between the set and the actual defocus taken as well as the point spread function at higher defocus values should be taken into account when setting the target defocus. 
20. Align movie frames e.g. with MotionCor2 program (30,31), WARP (32) or other software.

21. Estimate the CTF of the micrographs (e.g. Gctf (33), CTFfind (34), WARP (32)). Therefore, the approximate target defocus range (e.g. for $-500 \mathrm{~nm}$ set target defocus, search between -200 and $-1200 \mathrm{~nm}$ ) and phase shift $\left(\right.$ e.g. $\left.10-180^{\circ}\right)$ should be set in the search to restrict the program search, which will avoid misinterpretations of the program. Images taken with high phase shift can easily be interpreted as higher defocus images instead (see Figure 2B and C).

22. Plot the estimated phase shifts of the aligned movies (e.g. with Microsoft Excel) in the order the movies were taken. For each phase plate position the phase shift should build up gradually until it reaches a plateau as shown in Figure 2A (see Note 6). This can be done after the collection of $\sim 100$ images. The change of the phase plate position should be set to a time/number of exposures that corresponds to the time needed for the phase shift to reach a plateau at around $140^{\circ}$ (so that the phase shift range of the data lies between $90^{\circ}$ and $135^{\circ}$ ). Here the exact number of degrees is less important than the quality of the overall aligned images and the plateau-behaviour of the phase plate (see notes $7,8,9)$. The change of the phase plate position can be done automatically by an independent auto-clicking program (e.g. ClickWhen) or inside the data collection program (e.g. EPU (Thermo Fisher Scientific)) 


\section{Notes}

1. The condenser aperture can be enlarged to 100, if it is hard to see any features on the fluorescence screen. Additionally, the magnification can be increased momentarily. 2. Both modes, microprobe and nanoprobe, need to be aligned even if only one of the modes is used for data collection.

3. It may happen that this procedure changes the Diffraction Astigmatism. Therefore, one can reset the diffraction astigmatism in Diffraction mode (VPP retracted because of the strong crossover beam to protect the VPP) using the "Diffraction" box in the "Stigmator" tab.

4. Since data collection is rather close to focus, it is important to focus precisely to avoid massive data loss due to in-focus or over-focussed images.

5. It should be ensured that Thon rings are clearly visible in the Power spectra. If the signal of the images is so low that at $-500 \mathrm{~nm}$ defocus only one or no Thon rings are visible, the target defocus should be increased.

6. If the phase shift plot does not show a gradual increase of phase shift over time/exposure dose, it may indicate that either the phase shift estimation is not correct, the beam shift pivot pints on the VPP are not set correctly or the VPP is not in the backfocal plane; see steps described above.

7. Phase shifts around $135-180^{\circ}$ are more difficult to process because of image blurring. Phase shifts beyond $180^{\circ}$ an currently not be used in CTF correction. Also certain error proneness in the correctness of the programs reporting the phase shift needs to be considered. Therefore, it is advisable to consider the behaviour of the phase shift over time (Figure 2A) rather than the phase shift degree values. 
8. The accumulative dose for phase shift activation can change during ageing of the VPP. Therefore, it is advisable to monitor the phase shift behaviour during each data collection.

9. For tomography, data acquisition with a gradual phase shift increase is inconvenient. Therefore, the phase plate should be activated to a phase shift of $\sim 1 / 2 \pi\left(90^{\circ}\right)$ before the tilt series data collection is started (i.e. close to the above described plateau; this helps to have similar phase shift values within a tilt series).

10. Occasionally, small contaminations on the VPP induce a non-equal phase shift inside an image. These contaminations can be permanent or not (some may disappear during exposure to the electron beam).

11. The astigmatism induced by the VPP could vary in some pre-set positions on the VPP. It may be possible to exclude VPP positions with high astigmatism by scripting before data collection. 


\section{References}

1. Zernike F (1955) How I discovered phase contrast. Science 121:345-349

2. Herzik MA, Wu M, and Lander GC (2019) High-resolution structure determination of sub-100 kDa complexes using conventional cryo-EM. Nat Commun 10:1032

3. Orlov I, Rochel N, Moras D, et al (2012) Structure of the full human RXR/VDR nuclear receptor heterodimer complex with its DR3 target DNA. EMBO J 31:291300

4. Dubochet J, Adrian M, Chang JJ, et al (1988) Cryo-electron microscopy of vitrified specimens. Q Rev Biophys 21:129-228

5. Cambie R, Downing KH, Typke D, et al (2007) Design of a microfabricated, twoelectrode phase-contrast element suitable for electron microscopy. Ultramicroscopy 107:329-339

6. Danev R and Nagayama K (2001) Transmission electron microscopy with Zernike phase plate. Ultramicroscopy 88:243-252

7. Frindt N, Oster M, Hettler S, et al (2014) In-focus electrostatic Zach phase plate imaging for transmission electron microscopy with tunable phase contrast of frozen hydrated biological samples. Microsc Microanal Off J Microsc Soc Am Microbeam Anal Soc Microsc Soc Can 20:175-183

8. Majorovits E, Barton B, Schultheiss K, et al (2007) Optimizing phase contrast in transmission electron microscopy with an electrostatic (Boersch) phase plate. Ultramicroscopy 107:213-226

9. Walter A, Steltenkamp S, Schmitz S, et al (2015) Towards an optimum design for electrostatic phase plates. Ultramicroscopy 153:22-31

10. Danev R, Buijsse B, Khoshouei M, et al (2014) Volta potential phase plate for infocus phase contrast transmission electron microscopy. Proc Natl Acad Sci U S A 111:15635-15640

11. Danev R, Tegunov D, and Baumeister W (2017) Using the Volta phase plate with defocus for cryo-EM single particle analysis. Elife 6

12. Dai W, Fu C, Raytcheva D, et al (2013) Visualizing virus assembly intermediates inside marine cyanobacteria. Nature 502:707-710

13. Murata K, Liu X, Danev R, et al (2010) Zernike Phase Contrast Cryo-Electron Microscopy and Tomography for Structure Determination at Nanometer and SubNanometer Resolutions. Struct Lond Engl 1993 18:903-912

14. Li K, Sun C, Klose T, et al (2019) Sub-3 Å apoferritin structure determined with full range of phase shifts using a single position of volta phase plate. J Struct Biol 206:225-232

15. Fan X, Zhao L, Liu C, et al (2017) Near-Atomic Resolution Structure Determination in Over-Focus with Volta Phase Plate by Cs-Corrected Cryo-EM. Structure 25:1623-1630.e3

16. Loeffelholz O von, Papai G, Danev R, et al (2018) Volta phase plate data collection facilitates image processing and cryo-EM structure determination. $\mathrm{J}$ Struct Biol 202:191-199

17. Fan X, Wang J, Zhang X, et al (2018) Single particle cryo-EM reconstruction of $52 \mathrm{kDa}$ streptavidin at 3.2 Angstrom resolution. bioRxiv 457861 
18. Hill CH, Boreikaite V, Kumar A, et al (2019) Activation of the Endonuclease that Defines mRNA 3' Ends Requires Incorporation into an 8-Subunit Core Cleavage and Polyadenylation Factor Complex. Mol Cell 73:1217-1231.e11

19. Khoshouei M, Radjainia M, Baumeister W, et al (2017) Cryo-EM structure of haemoglobin at $3.2 \AA$ determined with the Volta phase plate. Nat Commun 8:16099

20. Abeyrathne PD, Koh CS, Grant T, et al (2016) Ensemble cryo-EM uncovers inchworm-like translocation of a viral IRES through the ribosome. Elife 5

21. Klaholz BP, Myasnikov AG, and Van Heel M (2004) Visualization of release factor 3 on the ribosome during termination of protein synthesis. Nature 427:862865

22. Penczek PA, Frank J, and Spahn CM (2006) A method of focused classification, based on the bootstrap 3D variance analysis, and its application to EF-G-dependent translocation. J Struct Biol 154:184-94

23. Scheres SH (2010) Classification of structural heterogeneity by maximumlikelihood methods. Methods Enzym 482:295-320

24. Loeffelholz O von, Natchiar SK, Djabeur N, et al (2017) Focused classification and refinement in high-resolution cryo-EM structural analysis of ribosome complexes. Curr Opin Struct Biol 46:140-148

25. Zivanov J, Nakane T, Forsberg BO, et al (2018) New tools for automated highresolution cryo-EM structure determination in RELION-3. eLife 7

26. Afonine PV, Klaholz BP, Moriarty NW, et al (2018) New tools for the analysis and validation of cryo-EM maps and atomic models. Acta Crystallogr Sect Struct Biol 74:814-840

27. Khoshouei M, Pfeffer S, Baumeister W, et al (2017) Subtomogram analysis using the Volta phase plate. J Struct Biol 197:94-101

28. Rast A, Schaffer M, Albert S, et al (2019) Biogenic regions of cyanobacterial thylakoids form contact sites with the plasma membrane. Nat Plants 5:436

29. Mastronarde DN (2005) Automated electron microscope tomography using robust prediction of specimen movements. J Struct Biol 152:36-51

30. Li X, Mooney P, Zheng S, et al (2013) Electron counting and beam-induced motion correction enable near-atomic-resolution single-particle cryo-EM. Nat Methods 10:584-90

31. Zheng SQ, Palovcak E, Armache JP, et al (2017) MotionCor2: anisotropic correction of beam-induced motion for improved cryo-electron microscopy. Nat Methods

32. Tegunov D and Cramer P (2018) Real-time cryo-EM data pre-processing with Warp. bioRxiv 338558

33. Zhang K (2016) Gctf: Real-time CTF determination and correction. J Struct Biol 193:1-12

34. Rohou A and Grigorieff N (2015) CTFFIND4: Fast and accurate defocus estimation from electron micrographs. J Struct Biol 192:216-21 


\section{Acknowledgments}

We thank Jonathan Michalon, Mathieu Schaeffer, Remy Fritz and Romaric David for IT support. This work was supported by CNRS, Association pour la Recherche sur le Cancer (ARC), Institut National du Cancer (INCa), the Fondation pour la Recherche Médicale (FRM), Ligue nationale contre le cancer (Ligue), Agence National pour la Recherche (ANR) and USIAS (USIAS-2018-012). The electron microscope facility was supported by the Alsace Region, FRM, Inserm, CNRS and ARC, the French Infrastructure for Integrated Structural Biology (FRISBI) ANR-10-INSB-05-01, by Instruct-ERIC and Instruct-ULTRA.

Correspondence and requests for materials should be addressed to B.P.K.

(klaholz@igbmc.fr). The authors declare no competing financial interests. 


\section{Figure legends}

\section{Figure 1: Placement of the VPP into the back-focal plane}

(a) Scheme of an electron microscope including the position of the VPP at the height of the objective apertures. Images show the artefacts visible when the VPP is not in the back-focal plane or when condenser astigmatism is present.

\section{Figure 2: Monitoring of the phase shift during VPP cryo-EM data acquisition}

(a) Phase shift development over time/exposure dose on a single phase plate position.

(b) Zoomed power spectrum-halves of images with a low phase shift (left) and a high phase shift (right) at the same defocus.

(c) Ribosome-images acquired at a similar defocus with the same dose of $30 \mathrm{e} / \AA^{2}$ containing a low phase shift (left) and a high phase shift (right).

(d) Shadows appearing in a cryo-EM image of human ribosomes collected with a VPP that was not inserted well in the back-focal plane.

(e,f) Cryo-EM image of human ribosomes collected with a contaminated VPP. 


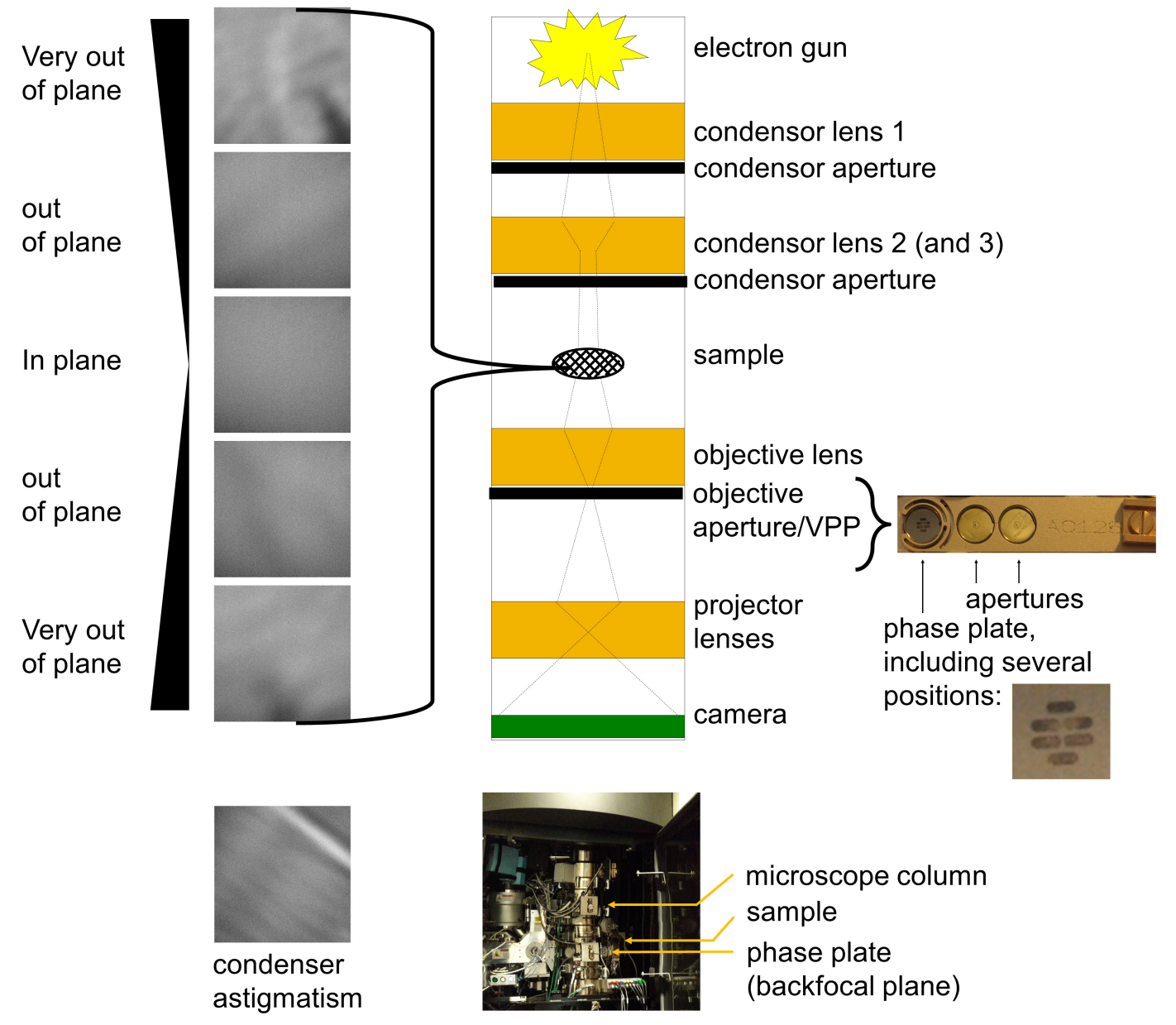


A

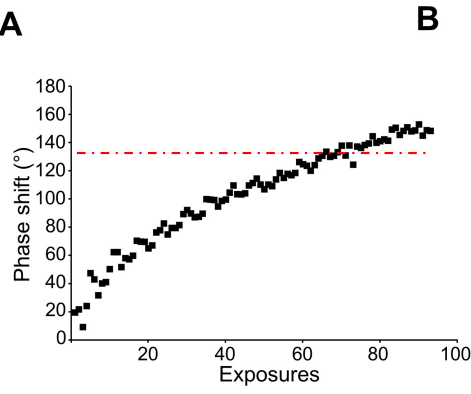

D

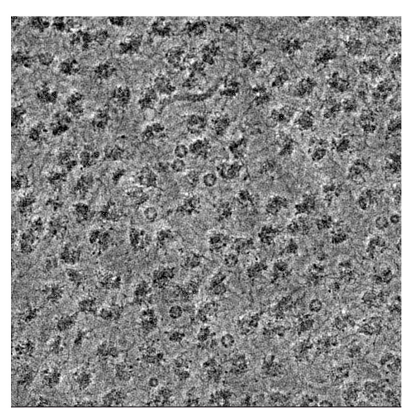

VPP not in back-focal plane

B

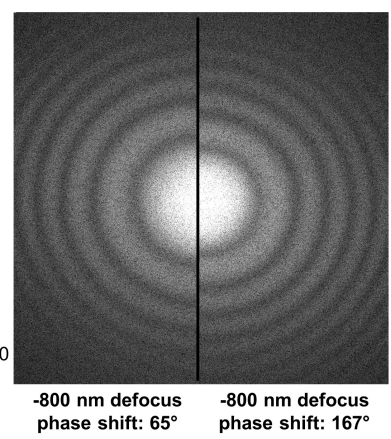

E

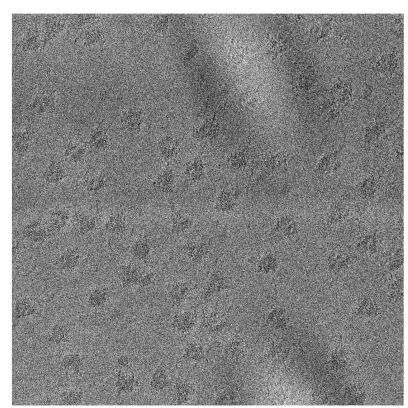

VPP with contamination

C

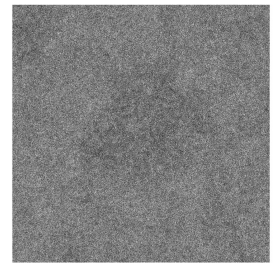

$-420 \mathrm{~nm}$ defocus

(gives weak contrast)

phase shift: $65^{\circ}$
(gives stronger contrast)

F

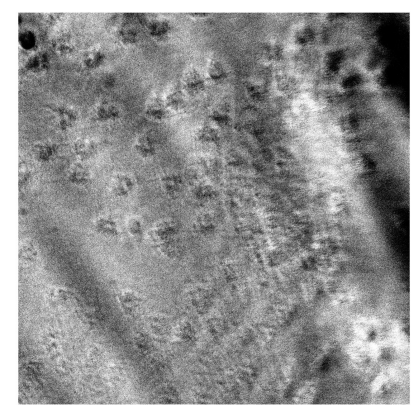

VPP with contamination 